\title{
Hitos sobre el test cardiopulmonar de ejercicio
}

\author{
RODRIGO SOTO F.*,**,*** e IVÁN CAVIEDES S.**,***
}

\section{Cardiopulmonary exercise test}

Cardiopulmonary exercise test (CPET) evaluates cardiorespiratory function in dynamic conditions, in contrast to static pulmonary function tests which can not reproduce the dynamic situation of an individual. Despite its complex implementation there has been growing interest in CPET in evaluating the mechanisms of dyspnoea With this respect dynamic flow/volume curve is useful in identifying expiratory airflow limitation and dynamic hyperinflation. Besides gas exchange analysis during exercise deepens the quality of information in that subject. Its utility for decision making in complex perioperative evaluation can not be overemphasized. Considering its high reproducibility, this method is a highly valuable tool in cardiac stress testing. Several publications in Cardiology emphasize its value in diagnosis, follow up, prognosis and enlisting for transplant in congestive heart failure. Remarkably useful are maximal and submaximal indices obtained during exercise, such as peak oxygen consumption among the maximals, and carbon dioxide equivalent and oxygen consumption at lactic threshold, among the submaximal indicators.

Key words: Cardiopulmonary exercise test, dynamic hyperinflation, perioperative evaluation, peak oxygen uptake.

\section{Resumen}

El test cardiopulmonar de ejercicio TCPE (CPET en la literatura anglosajona) evalúa aspectos dinámicos de la la fisiología cardiorespiratoria, en contraste, las pruebas funcionales estáticas no reproducen la condición activa de un individuo. No obstante su complejo montaje e interpretación, en el último decenio ha habido un creciente interés en aplicarlo en la detección de mecanismos de disnea. Al respecto, la curva flujo/volumen dinámica permite demostrar limitación de flujos e hiperinflación dinámica, también esta prueba permite profundizar en el análisis del intercambio gaseoso en ejercicio. La utilidad de estas pruebas en la evaluación funcional pre-operatoria compleja es destacada. Dada su alta reproducibilidad, el TCPE continúa siendo un alto referente en pruebas cardíacas de esfuerzo. Publicaciones cardiológicas enfatizan su valor pronóstico como indicador de sobrevida, seguimiento de terapias y enlistado para trasplante en insuficiencia cardíaca congestiva, utilizando parámetros máximos y submáximos. Entre los primeros el consumo cumbre o punta de oxígeno, y en los submáximos el equivalente ventilatorio de anhídrido carbónico y el consumo de oxígeno en el umbral láctico.

Palabras clave: Test cardiopulmonar de ejercicio; hiperinflación dinámica; evaluación prequirúrgica; consumo de $\mathrm{O}_{2}$ máximo.

\section{Introducción}

Condición básica de la vida diaria es la capacidad de efectuar trabajo aeróbico, que requiere de la integración respiratoria, hematológica,

cardiovascular y muscular. La posibilidad de evaluar el consumo de oxígeno cumbre o punta $\left(\mathrm{VO}_{2} \max \right)$ ha aportado importante información diagnóstica y pronóstica en una amplia variedad de condiciones clínicas, entre ellas la evaluación

\footnotetext{
* Unidad de Cuidados Intensivos y Servicio de Respiratorio, Hospital Fuerza Aérea de Chile.

** Servicio y Laboratorio Broncopulmonar, Clínica Alemana de Santiago.

*** Facultad de Medicina Clínica Alemana-Universidad del Desarrollo.
} 
de pacientes con patología cardiovascular, la identificación de mecanismos que condicionan disnea y que resultan limitantes para el ejercicio, la planificación de rehabilitación, la evaluación pre quirúrgica y el estudio de los candidatos a trasplante cardíaco, entre otras.

En el último decenio el test cardiopulmonar de ejercicio (TCPE) ha generado avances en diversas áreas de particular interés para los médicos clínicos, tres de las cuales se destacarán en este artículo, de carácter esencialmente didáctico:

1. La curva flujo/volumen dinámica en ejercicio como elemento de detección y seguimiento de mecanismos que contribuyen a la generación de disnea.

2. La utilidad del TCPE en la evaluación funcional previa a cirugía de resección pulmonar.

3. La utilidad del TCPE en el seguimiento de la insuficiencia cardíaca y enlistado de pacientes que requieren trasplante cardíaco.

\section{Curva flujo volumen dinámica como elemento de detección y seguimiento de los mecanismos de la disnea}

En el paciente con EPOC las alteraciones de intercambio gaseoso en ejercicio así como también el fenómeno de alteración de la distensibilidad dependiente de la frecuencia son relevantes en la limitación de la tolerancia al esfuerzo, su descripción escapa del objetivo de este análisis.

Por su parte, la sobreposición del trazado flujo/ volumen durante el ejercicio, dentro de la curva flujo/volumen máxima basal del paciente, permite detectar patrones de limitación de flujos espiratorios e hiperinflación dinámica en actividad física. Las publicaciones de Johnson y $\mathrm{O}^{\prime}$ Donnell ${ }^{1-3}$, se han constituido en referentes del tema. Estos autores hacen hincapié en dos factores que pueden limitar la tolerancia al ejercicio:

\section{1) Limitación de los flujos espiratorios:}

Es normal que el flujo espiratorio de una persona en ejercicio se vea limitado en hasta el $25 \%$ del proceso espiratorio, esto ocurre particularmente al llegar a volúmenes pulmonares bajos. Una limitación superior a este $25 \%$ constituye evidencia de limitación anormal de flujos para ventilar durante el ejercicio (Figura 1), su presencia puede llegar a generar una espiración incompleta, y consecuentemente un aumento del volumen pulmonar al final de la espiración, o Capacidad Residual Funcional dinámica. Este efecto se acentúa cuando existe taquipnea, dado que en ésta se acorta el tiempo espiratorio.

\section{2) Hiperinflación Dinámica (HD):}

Significa atrapamiento aéreo durante el ejercicio, deriva de la espiración incompleta generada por la limitación de los flujos espiratorios. Frente a la necesidad de incrementar el volumen minuto, el paciente no alcanza a espirar. La consecuencia es un aumento del volumen de fin de espiración, o capacidad residual funcional dinámica (VFE o CRFd).

Recíprocamente se genera una disminución de la capacidad inspiratoria (CI) a expensas de sus dos componentes, el volumen corriente $(\mathrm{Vt})$ y principalmente el volumen de reserva inspiratorio $(\mathrm{VRI})^{3}$. En definitiva el VFE queda determinado dinámicamente por dos factores: la limitación de flujos espiratorios, y la demanda ventilatoria.

En individuos sanos el volumen corriente aumenta durante el ejercicio a expensas tanto del volumen de reserva inspiratorio como del volumen de reserva espiratorio, en ellos la estrategia ventilatoria o volumen operacional se enmarca dentro del segmento escarpado de la curva presión/volumen, en donde la distensibilidad toracopulmonar es mayor. En cambio, en los pacientes con enfermedad pulmonar obstructiva crónica (EPOC), el incremento del volumen corriente en ejercicio, limitado por el atrapamiento gaseoso, se produce más bien a expensas del Volumen de Reserva Inspiratoria, desplazando el volumen operacional ventilatorio hacia arriba y hacia la derecha de la curva presión/volumen.

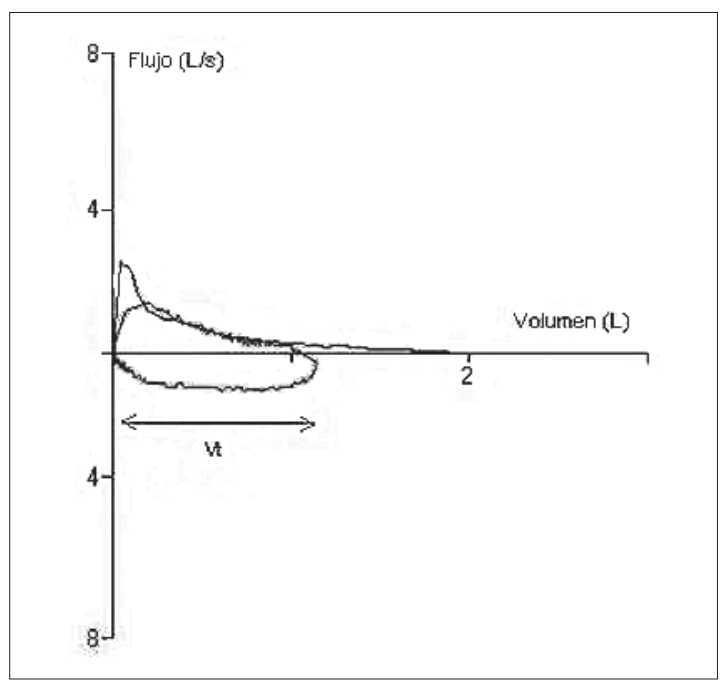

Figura 1. Limitación de flujos anormal, $>25 \%$ de la espiración. $\mathrm{Vt}$ = volumen corriente. 
En estas condiciones el paciente ventila en un régimen o volumen operacional más alto en esta curva, situando su respiración en una condición mecánica toracopulmonar menos complaciente, lo que asociado al acortamiento consiguiente de los músculos inspiratorios, genera disnea por aumento de la carga viscosoelástica para ventilar, y desventaja muscular inspiratoria.

Son manifestaciones de HD en ejercicio en pacientes con EPOC: un volumen final de inspiración (VFI) elevado, sobre el $85 \%$ de la capacidad pulmonar total (CPT), y a una disminución de la $\mathrm{CI}$, a expensas principalmente de una severa disminución del VRI (Figura 2).

En un universo de 105 pacientes con EPOC, O`Donnell ${ }^{2}$ detectó que el $80 \%$ de ellos experimentó HD, con una media de disminución de la CI durante el ejercicio del 14\%, lo que corresponde a una media de volumen de $370 \mathrm{ml}$. En otro estudio de 29 pacientes con EPOC, el mismo autor ${ }^{4}$ describió el efecto favorable del bromuro de ipratropio sobre la variación de la CI y su estrecha correlación con el alivio de la disnea medida según la escala de Borg. Belman, utilizando la relación VFI/CPT en ejercicio submáximo, demostró relación entre la disminución de la hiperinflación dinámica y el alivio de la disnea según la escala de Borg, al administrar Salbuta$\mathrm{mol}^{5}$.

En otras acciones médicas en las que se ha actuado sobre el volumen operacional de la ventilación, tal como la cirugía de reducción de volumen pulmonar, se ha demostrado reducción de la disnea (según la escala de Borg), en ejercicio submáximo, objetivándose disminución de la relación VFE/CPT en el mismo tiempo (o isotiempo) de un ejercicio submáximo, comparan-

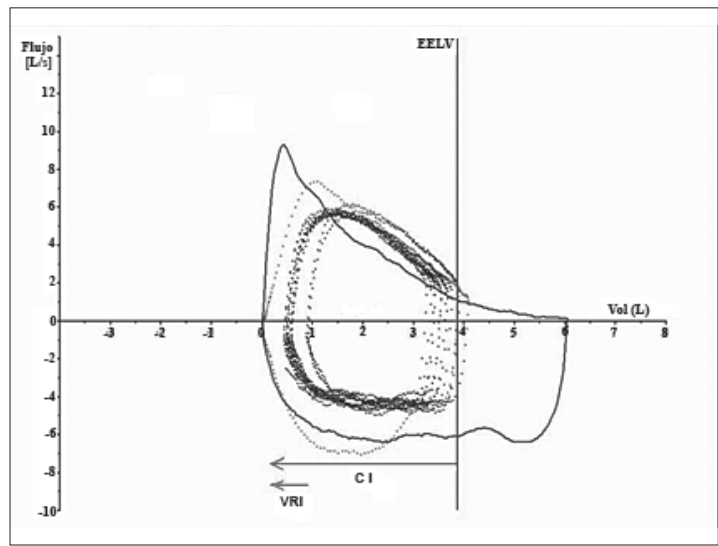

Figura 2. Curva flujo/volumen dinámica. La flecha CI indica capacidad inspiratoria, la flecha VRI indica el volumen de reserva inspiratoria. EELV = volumen de fin de espiración. do las pruebas pre y post operatorias. La disminución de la disnea se derivaría de un menor nivel de hiperinflación dinámica en el ejercicio ${ }^{6,7}$. Por su parte, la administración de oxígeno, que reduce la ventilación minuto submáxima en ejercicio, permite retrasar la generación de taquipnea y por ende hiperinflación dinámica, y ha demostrado prolongar la capacidad de sostener un esfuerzo submáximo ${ }^{8}$, constituyéndose así en un pilar irrenunciable de la práctica de programas de rehabilitación con ejercicio para pacientes con EPOC.

Al analizar la información de esta manera, se puede afirmar que cambios funcionales que podrían tener mínima o nula expresión en el volumen espiratorio forzado en el primer segundo $\left(\mathrm{VEF}_{1}\right)$, sí tienen impacto cuantificable si se determinan con pruebas de ejercicio submáximo, especialmente en isotiempos definidos, para tabular la respuesta frente a acciones terapéuticas, tales como el uso de fármacos y rehabilitación. Su expresión más concreta es la curva flujo/volumen dinámica, mediante la determinación de la CI, VRI y relaciones Vt/CI, VFI/CPT y VFE/CPT.

Estos conceptos suponen el origen de mejores indicadores pronósticos en EPOC al efectuar estudios dinámicos, considerando el valor limitado del $\mathrm{VEF}_{1}$ como determinante pronóstico en esta patología ${ }^{9,10}$.

\section{Utilidad del TCPE en la evaluación funcional previa a cirugía de resección pulmonar}

Es preciso hacer consideraciones sobre la reserva funcional de un paciente candidato a cirugía resectiva pulmonar, debido a que pueden presentarse los siguientes escenarios.

1. La posibilidad de generar una invalidez respiratoria.

2. El riesgo de efectuar una cirugía y terapia insuficiente como resultado de una mala reserva funcional.

3. La expectativa de complicaciones cardiopulmonares durante el postoperatorio.

Históricamente las complicaciones más temidas de resección pulmonar han sido la hipoventilación alveolar aguda, las arritmias cardíacas, el tromboembolismo pulmonar, el infarto del miocardio, las atelectasias lobares o bien la ventilación mecánica prolongada. Hoy todas estas tienen mayor opción de tratamiento exitoso en las unidades de cuidados intensivos. Por su parte Beckles y cols, han afirmado que el pronósti- 
co vital de los pacientes con cáncer pulmonar inoperable según criterios funcionales, no se modifica si el paciente es operado o no ${ }^{11}$.

La utilidad de la evaluación funcional pre operatoria con pruebas dinámicas no es un concepto nuevo, nació con los tests de ascenso de escaleras, en boga hace más de tres décadas. Van Nostrand ${ }^{12}$, reportó una mortalidad post quirúrgica del $50 \%$ si un paciente no era capaz de subir 2 pisos de escalera, cifra que caía a un $11 \%$ si el paciente era capaz de ascender esta distancia. En otro estudio, Olsen ${ }^{13}$, reportó un acentuado aumento de las complicaciones post operatorias si el paciente subía menos de 2 pisos, y Girish ${ }^{14}$, por su parte, determinó un valor predictivo positivo de $82 \%$ para presentar complicaciones post operatorias, si el paciente ascendía menos de 2 pisos de escalera.

En la decisión quirúrgica el TCPE tiene un rol definido y consolidado. Mediante pruebas de ejercicio se puede estimar con precisión la morbilidad quirúrgica esperada ${ }^{15}$. Publicaciones cardiológicas han establecido como punto de corte una capacidad de ejercicio de 5 mets, que corresponde aproximadamente a $17,5 \mathrm{ml} / \mathrm{kg}$ de $\mathrm{VO}_{2} \max$, como un límite bajo el cual hay una alta tasa de complicaciones, entre ellas los infartos del miocardio en pacientes coronarios.

Producto de la revisión de 14 series clínicas, Bolliger ${ }^{16}$, definió un algoritmo para la selección de pacientes con pruebas de función pulmonar estática severamente alteradas que son candidatos a resección pulmonar (Figura 3). En éstos pacientes el autor estimó el $\mathrm{VO}_{2} \max$ post operatorio predicho, multiplicando el $\mathrm{VO}_{2} \max$ medido, por el porcentaje de tejido pulmonar remanente predicho, determinado por cintigrafía de perfusión pulmonar cuantitativa, y postuló que la cirugía resectiva pulmonar es practicable si el $\mathrm{VO}_{2} \max$ postoperatorio predicho resulta igual o mayor a $10 \mathrm{ml} / \mathrm{kg} / \mathrm{min}$, (o $35 \%$ del $\mathrm{VO}_{2}$ max teórico normal). Un criterio semejante aunque no idéntico, es propuesto en el Consenso Nacional en $\mathrm{EPOC}^{17}$.

El mismo autor ${ }^{16,18}$ aplicó este protocolo en 80 pacientes, destacando que los pacientes que habían fallecido, pertenecían al grupo que presentaba un $\mathrm{VO}_{2} \max$ menor al $60 \%$ del valor teórico máximo. Entre 9 pacientes con esta condición, 8 presentaron complicaciones y 3 falle-

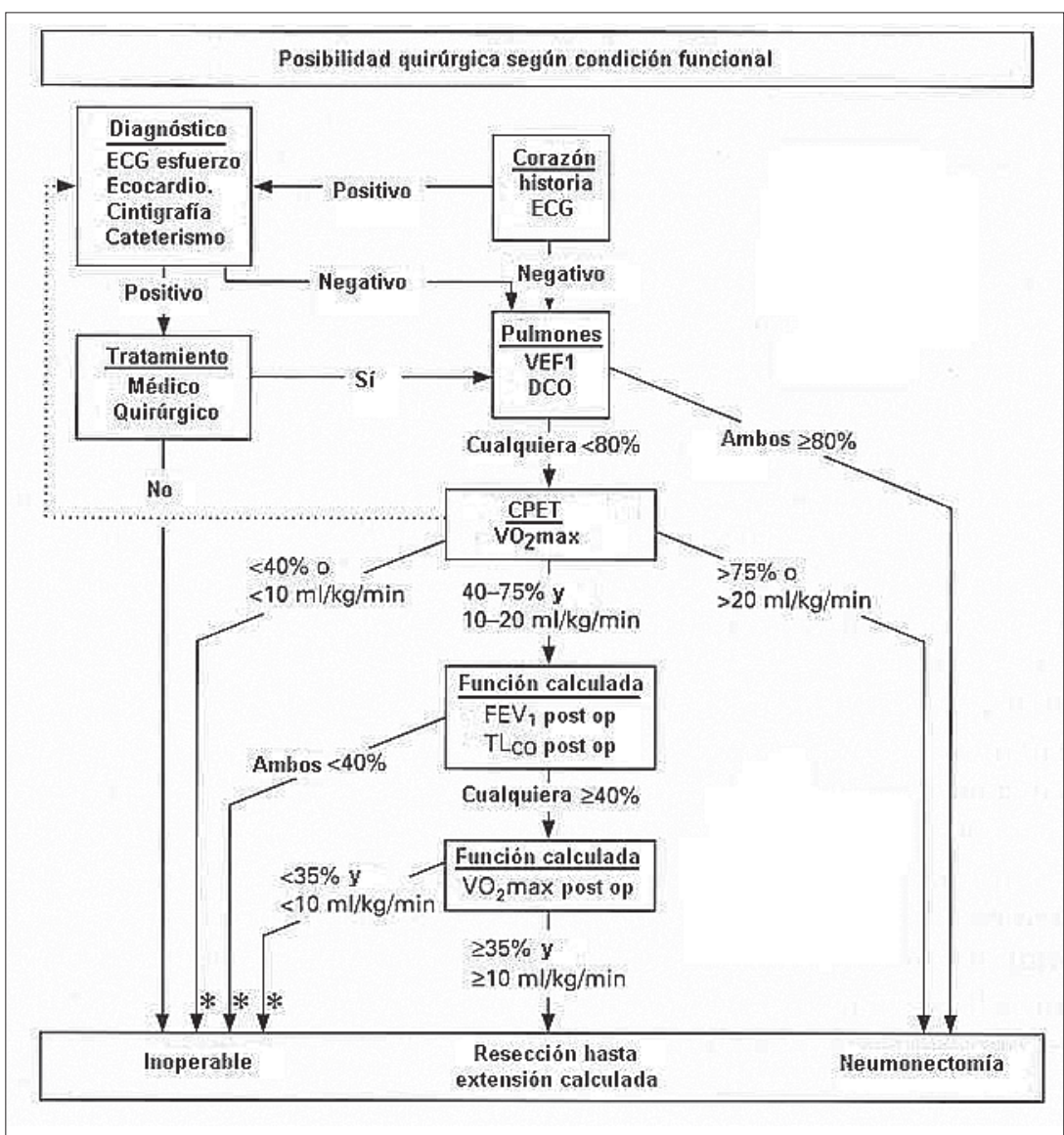

Figura 3. Algoritmo de selección de pacientes para cirugía de resección pulmonar (Adaptado de Bolliger C. et $\mathrm{al}^{16}$ ). 
cieron. Wyser ${ }^{19}$, utilizó este protocolo en 137 pacientes candidatos a cirugía resectiva pulmonar, destacando que la mortalidad se redujo de 4 a $1,5 \%$, y las complicaciones de 20 a $11 \%$. Logró además recuperar para tratamiento quirúrgico, algunos pacientes que por pruebas de función pulmonar estática habrían sido considerados inoperables.

\section{Utilidad del TCPE en el seguimiento de la insuficiencia cardíaca y en la definición de los pacientes que requieren trasplante cardíaco}

$\boldsymbol{V O}_{2}$ max: La determinación del $\mathrm{VO}_{2}$ max tiene mejor correlación con la capacidad funcional clínica que las mediciones clásicas de función cardíaca, tales como el gasto cardíaco, la fracción de eyección medida por ecocardiograma, la presión capilar pulmonar y otras. Es así como se han generado tablas que establecen un paralelo entre la clasificación clínica de la insuficiencia cardíaca de la New York Heart Association (NYHA), en clases de I a IV, confrontadas con la medición del consumo máximo de $\mathrm{O}_{2}$ en un TCPE (Tabla 1) ${ }^{20}$. El nivel de corte que predice una elevada mortalidad a 1 año, es un $\mathrm{VO}_{2} \max$ de $14 \mathrm{ml} / \mathrm{kg}$, esta cifra ha sido adoptada como el criterio central para enlistado de trasplante cardíaco $^{21-23}$ (Figura 4). Expresado en relación al valor predicho, cuando el $\mathrm{VO}_{2}$ max es inferior al 50\% de su teórico, se pueden extraer conclusiones semejantes.
Stevenson, demostró la utilidad del $\mathrm{VO}_{2} \max$ en la evaluación y el seguimiento de la terapia en pacientes con insuficiencia cardíaca congestiva (ICC). La autora intervino realizando ajustes de la terapia médica y rehabilitación, y observó mejoría del $\mathrm{VO}_{2} \max$ en un grupo de pacientes, que incluso superaron valores de corte sobre $14 \mathrm{ml} /$ $\mathrm{kg}$. Estos pacientes fueron retirados de la lista de trasplante cardíaco, y su evolución se asimiló consecuentemente a la del grupo que originalmente no requería trasplante ${ }^{24}$.

$\mathrm{VO}_{2}$ max y betabloqueo: El uso de bloqueadores de receptores adrenérgicos $\beta$ no modifica el $\mathrm{VO}_{2} \max$, sin embargo, su empleo disminuye la mortalidad esperada, haciendo dudar que el nivel de corte crítico para el grupo sea

Tabla 1. Gradación de diferentes niveles de deterioro de la capacidad aeróbica y del umbral anaeróbico, durante TCPE incremental

\begin{tabular}{clrc}
\hline Clase & Severidad & $\begin{array}{c}\mathbf{V O}_{2} \mathbf{m a x} \\
(\mathbf{m l} / \mathbf{m i n} / \mathbf{k g})\end{array}$ & $\begin{array}{c}\text { UL } \\
(\mathbf{m l} / \mathbf{m i n} / \mathbf{k g})\end{array}$ \\
\hline A & Leve & $>20$ & $>14$ \\
B & $\begin{array}{l}\text { Leve a } \\
\text { moderada }\end{array}$ & $16-20$ & $11-14$ \\
C & $\begin{array}{l}\text { Moderada a } \\
\text { severa }\end{array}$ & $10-16$ & $8-11$ \\
D & Severa & $6-10$ & $5-8$ \\
E & Muy severa & $<6$ & $<4$ \\
\hline
\end{tabular}

UL: umbral anaeróbico; $\mathrm{VO}_{2} \max$ : máximo consumo de oxígeno (adaptado de Tjahja J E, et $\mathrm{al}^{21}$ ).

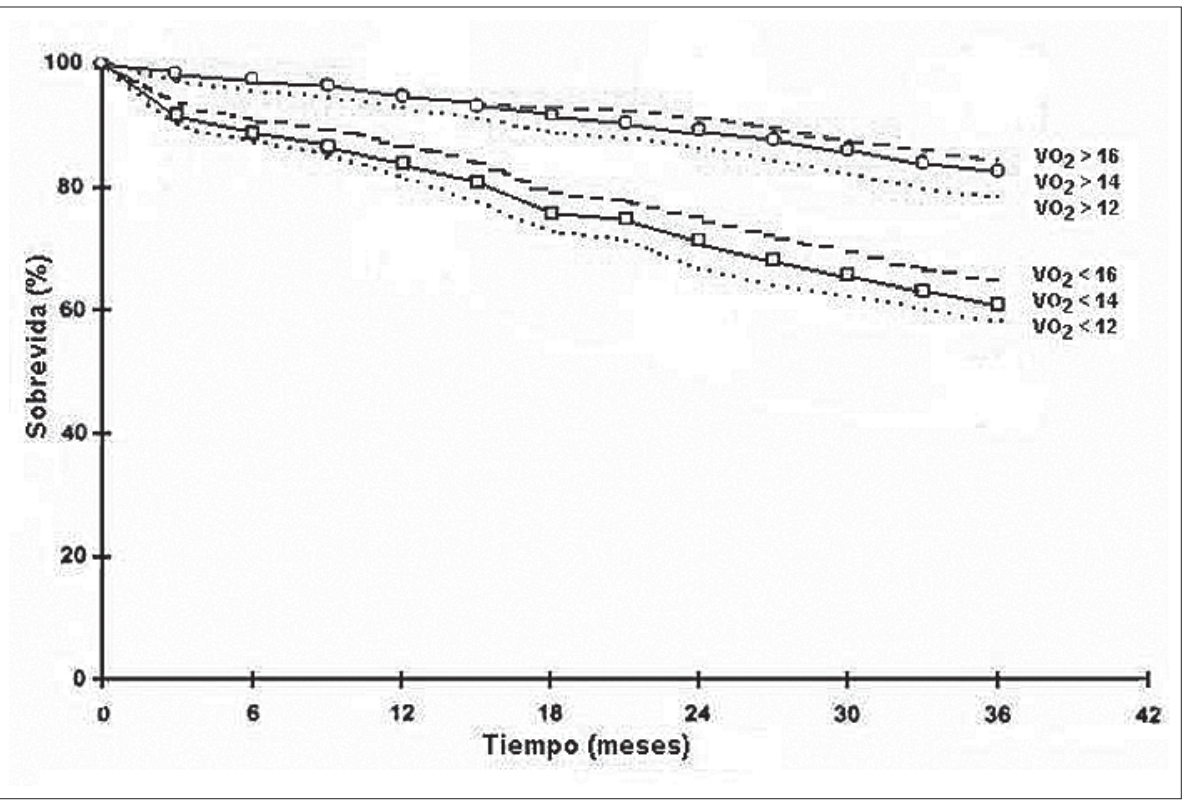

Figura 4. $\mathrm{VO}_{2} \max$ como índice pronóstico en insuficiencia cardíaca congestiva, grupos con $\mathrm{VO}_{2} \max$ mayor $\mathrm{y}$ menor de $14 \mathrm{ml} / \mathrm{kg}$ (adaptado de Myers J. et $\left.\mathrm{al}^{22}\right)$. 


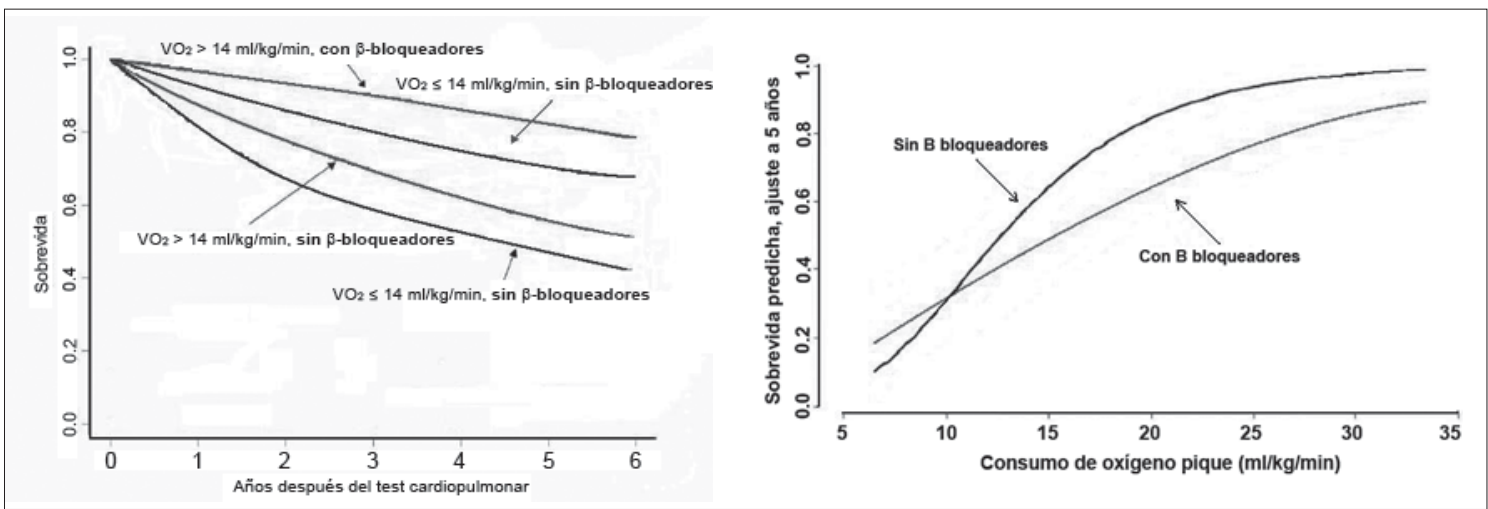

Figura 5. Niveles de $\mathrm{VO}_{2} \max$ y mortalidad en pacientes con y sin bloqueo de receptores adrenérgicos beta (adaptado de O'Neill J, et $\mathrm{al}^{25}$ )

$14 \mathrm{ml} / \mathrm{kg}$ de $\mathrm{VO}_{2} \max$ (Figura 5 a y b). En estos pacientes se observa que recién bajo niveles de $10 \mathrm{ml} / \mathrm{kg}$, aumenta significativamente la mortali$\operatorname{dad}^{24,25}$.

\section{Mediciones submáximas:}

$\boldsymbol{V O}_{2} \boldsymbol{U} \boldsymbol{L}:$ Fleg y $\operatorname{cols}^{26}$, han postulado que existe ventaja en medir el consumo de oxígeno a nivel submáximo, a nivel de umbral láctico $\left(\mathrm{VO}_{2} \mathrm{UL}\right)$, considerando que el $\mathrm{VO}_{2} \max$ es una determinación esfuerzo-voluntad dependiente, y no así la medición a nivel de umbral láctico. Esta medición submáxima correspondería a un valor más reproducible del nivel de insuficiencia cardíaca congestiva. En este contexto Itoh $^{27}$, demostró que existe una buena correlación entre el $\mathrm{VO}_{2} \mathrm{UL}$ y la clasificación funcional clínica de insuficiencia cardíaca de la NYHA (Tabla 2). El nivel de corte propuesto para enlistado utilizando este parámetro es $\mathrm{VO}_{2} \mathrm{UL}<11 \mathrm{ml} / \mathrm{kg}$.

Equivalentes de $\mathrm{CO}_{2}$ : La eficiencia ventilatoria para eliminar $1 \mathrm{~L}$ de $\mathrm{CO}_{2}$, o Equivalente ventilatorio de $\mathrm{CO}_{2}$ a nivel de umbral láctico $\left(\mathrm{EqCO}_{2}\right.$, (Figura 6), es otra medición independiente de la voluntad, pues se realiza en ejercicio submáximo, y ha demostrado tener un fuerte valor predictivo positivo para mortalidad y para la necesidad de hospitalización en pacientes con

Tabla 2. Correlación entre $\mathrm{VO}_{2} \mathrm{UL}$ con la clasificación funcional clínica de insuficiencia cardíaca de la NYHA

$\begin{array}{ll}\mathrm{VO}_{2} \mathrm{UL} \text { 90\% del teórico normal } & \text { Clase I } \\ \mathrm{VO}_{2} \text { UL 77\% del teórico normal } & \text { Clase II } \\ \mathrm{VO}_{2} \text { UL 60\% del teórico normal } & \text { Clase III }\end{array}$

$\mathrm{VO}_{2} \mathrm{UL}$ : consumo de oxígeno máximo a nivel del umbral láctico (adaptado de Itoh H. et $\mathrm{al}^{27}$ ) insuficiencia cardíaca congestiva (ICC). Este dato también puede variar frente a ajustes favorables del tratamiento farmacológico o rehabilitación exitosa en ICC. Una limitación de esta medición es que puede verse alterada en casos de hiperventilación o hipercapnia, de modo que puede ser necesario confrontarla con la determinación de gases arteriales. En contraste, la medición de $\mathrm{VO}_{2} \max$ no requiere de punción arterial.

El nivel de corte para el $\mathrm{EqCO}_{2}$, es igual o menor a 34 litros de ventilación por litro de $\mathrm{CO}_{2}$, y tiene una sensibilidad y especificidad de $73 \%$ y $67 \%$ respectivamente, para la predicción de hospitalizaciones durante el transcurso de 1 año, esta información resulta relevante para ajustar tratamientos y planificar empleo de recursos. El estudio de Arena ${ }^{28}$, con 2.015 pacientes, ha puesto de manifiesto el aporte pronósti-

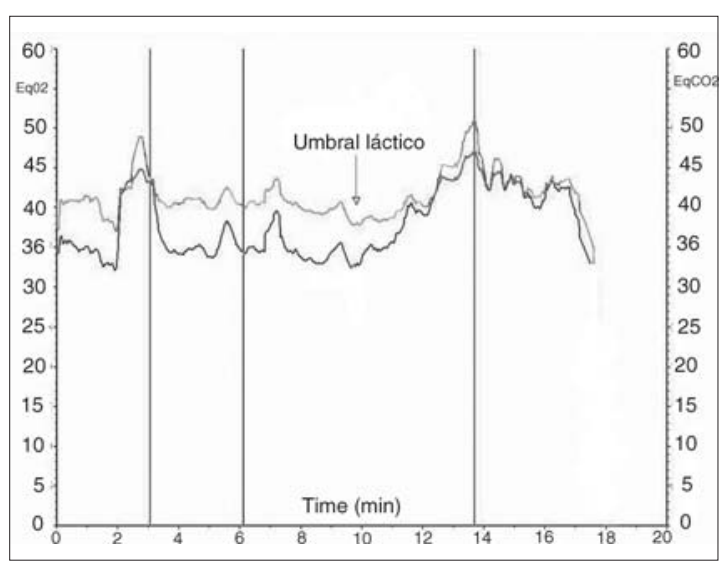

Figura 6. Curvas de equivalentes ventilatorios de $\mathrm{O}_{2}$ y $\mathrm{CO}_{2}$. Con línea gris se indica el equivalente ventilatorio de $\mathrm{CO}_{2}$, en este caso aumentado a nivel del umbral láctico $( \pm 38)$, representando ventilación ineficiente. $\mathrm{EqCO}_{2}$ = equivalente ventilatorio de $\mathrm{O}_{2}$ línea negra inferior. 
co del $\mathrm{EqCO}_{2}$ en pacientes con ICC, incluso superando el valor del $\mathrm{VO}_{2} \max$.

Este $\mathrm{EqCO}_{2}$ o $\mathrm{VE} / \mathrm{VCO}_{2}$, que refleja la eficiencia de la ventilación y se refiere a la ventilación del espacio muerto físiológico representado por la relación $\mathrm{Vd} / \mathrm{Vt}$ siendo $\mathrm{Vd}$ el espacio muerto y Vt el volumen corriente. Su alteración está relacionada con mala perfusión en la red capilar pulmonar, ya sea porque existe patología vascular pulmonar intrínseca o por bajo débito cardíaco. Un tercer factor es la pérdida de la distensibilidad pulmonar, que genera un patrón ventilatorio de volúmenes corrientes pequeños y frecuencias respiratorias altas, aumentando la representación del espacio muerto anatómico. En pacientes con EPOC, la elevación de la relación $\mathrm{Vd} / \mathrm{Vt}$ en ejercicio responde a una combinación de lo expuesto más un componente derivado de la hiperinflación.

Complementariamente, Chua y cols ${ }^{29}$, han reportado una sobrevida de $95 \%$ a 1 año en pacientes con ICC y $\mathrm{EqCO}_{2}$ menor a 34, y de $69 \%$ en aquellos con $\mathrm{EqCO}_{2}$ por sobre ese nivel.

Gitt, Wassermann y cols ${ }^{30}$, publicaron un seguimiento por 644 días a 223 pacientes con cardiopatía dilatada, y establecieron que el uso de los dos criterios combinados, $\mathrm{EqCO}_{2}>34$ y $\mathrm{VO}_{2} \mathrm{UL}<11 \mathrm{ml} / \mathrm{kg}$, permite estimar un riesgo relativo que duplica al criterio clásico de $\mathrm{VO}_{2} \max$ $<14 \mathrm{ml} / \mathrm{kg}$, independiente del sexo, edad, fracción de eyección o clase funcional.

El riesgo de la realización del TCPE es igual al de las pruebas de esfuerzo, esto es del orden de 2 incidentes fatales por cada 100.000 exámenes.

La puesta en marcha de esta prueba supone una inversión inicial importante en lo económico y en el desarrollo conceptual para su ejecución, de allí que no sea en nuestro medio una prueba de realización rutinaria.

La variabilidad de los valores que se reportan es del orden de $10 \%$ si se contemplan las recomendaciones de calibración con gases y calibración biológica, hechos cuyo análisis escapa del objetivo de este artículo, y pueden ser hallados en literatura de la referencia ${ }^{31}$.

\section{Conclusiones}

1) La adición de la curva flujo/volumen dinámica al TCPE, ha permitido comprender y medir factores mecánicos que limitan la tolerancia al ejercicio, como la limitación de flujos y la hiperinflación dinámica.
2) La realización de TCPE submáximos con curva flujo/volumen dinámica, permite detectar cambios ventilatorios en ejercicio que son relevantes, que impactan la sintomatología del paciente, que la espirometría no es capaz de demostrar, y resultan de interés en el control de tratamientos.

3) El TCPE es un instrumento de apoyo importante en la toma de decisiones para cirugía en general, y resectiva pulmonar en particular, en pacientes con patología respiratoria de base.

4) El TCPE es una eficiente herramienta en la clasificación y seguimiento de pacientes con insuficiencia cardíaca congestiva, tanto para la evaluación y planificación del tratamiento, como para la definición cuantitativa de los candidatos a trasplante cardíaco.

5) Existe interés creciente en el desarrollo de pruebas de ejercicio submáximas, para definir su aporte en el diagnóstico, tratamiento y control de pacientes con patología respiratoria y cardíaca.

6) Si bien el montaje del TCPE no es simple, su ejecución es factible en laboratorios de función pulmonar de referencia, con personal médico adiestrado y el equipamiento e implementación de reanimación adecuada, con un nivel de riesgo razonable y asumido desde las pruebas de esfuerzo convencionales.

\section{Bibliografía}

1.- JOHNSON B D, WEISMAN I M, ZEBALLOS R J, BECK K C. Emerging concepts in the evaluation of ventilatory limitation during exercise: The exercise tidal flow-volume loop. Chest 1999; 116: 488-503.

2.- O'DONNELL D E, REVILL S M, WEBB K A. Dynamic hyperinflation and exercise intolerance in chronic obstructive pulmonary disease. Am J Respir Crit Care Med 2001; 164: 770-7.

3.- O'DONNELL D E, BERTLEY J C, CHAU L K, WEBB K A. Qualitative aspects of exertional breathlessness in chronic airflow limitation: pathophysiologic mechanisms. Am J Respir Crit Care Med 1997; 155: 109-15.

4.- O’DONNELL D E. Assessment of bronchodilator efficacy in symptomatic COPD: is spirometry useful? Chest 2000; 117 (2 Suppl): 42S-7S.

5.- BELMAN M J, BOTNICK W C, SHIN J W. Inhaled bronchodilators reduce dynamic hyperinflation during exercise in patients with chronic obstructive pulmonary disease. Am J Respir Crit Care Med 1996; 153: 967. 75

6.- MARTÍNEZ F J, DE OCA M M, WHYTE R I, STETZ J, GAY S E, CELLI B R. Lung volume reduction improves dyspnea, dynamic hyperinflation and respiratory muscle function. Am J Respir Crit Care Med 1997; 155: 1984-90.

7.- O'DONNELL D E, WEBB K A, BERTLEY J C, CHAU L K, CONLAN A. A Mechanisms of relief of exertional 
breathlessness following unilateral bullectomy and lung volume reduction surgery in emphysema. Chest 1996: 110: $18-27$.

8.- O’DONNELL D E, D’ARSIGNY C, WEBB K A. Effects of hyperoxia on ventilatory limitation during exercise in advanced chronic obstructive pulmonary disease. Am J Respir Crit Care Med 2001; 163: 892-8.

9.- CELLI B R, COTE C G, MARÍN J M, CASANOVA C, MONTES DE OCA M, MÉNDEZ R A, et al. The body mass index, airflow obstruction, dyspnea, and exercise capacity index in chronic obstructive pulmonary disease. N Engl J Med 2004; 350: 100512.

10.- CASANOVA C, COTE C, DE TORRES J P, AGUIRREJAIME A, MARÍN J, PINTO PLATA V, et al. Inspiratory to total lung capacity ratio predicts mortality in patients with chronic obstructive pulmonary disease. Am J Respir Crit Care Med 2005; 171: 591-7.

11.- BECKLES M, SPIRO S, COLICE G, BECKLES M A, SPIRO S G, COLICE G L, et al. The physiologic evaluation of patients with lung cancer being considered for resectional surgery. Chest 2003; 123: 105S-14S.

12.- VAN NOSTRAND D, KJELSBERG M O, HUMPHREY E W. Preresectional evaluation of risk from pneumonectomy. Surg Gyn Obst 1968; 127: 306-12.

13.- OLSEN G N, BOLTON J W, WEIMAN D S, HORNUNG C A. Stair climbing as an exercise test to predict the postoperative complications of lung resection. Two years experience. Chest 1991; 99: 58790.

14.- GIRISH M, TRAYNER E J R, DAMMANN O, PINTO-PLATA V, CELLI B. Symptom-Limited Stair Climbing as a Predictor of Postoperative Cardiopulmonary Complications After High-Risk Surgery. Chest 2001; 120: 1147-51.

15.- REILLY J J Jr. Evidence-Based Preoperative Evaluation of Candidates for Thoracotomy. Chest 1999; 116: 474S-6S.

16.- BOLLIGER C T, PERRUCHOUD A P. Functional evaluation of the lung resection candidate. Eur Respir J 1998; 11: 198-212.

17.- LISBOA C, BORZONE G, CRUZ E. Inspiratory muscle training in patients with chronic obstructive pulmonary disease. Rev Méd Chile 1998; 126: 563-8.

18.- BOLLIGER C T, JORDAN P, SOLÈR M, STULZ P, GRÄDEL E, SKARVAN K, et al. Exercise capacity as a predictor of postoperative complications in lung resection candidates. Am J Respir Crit Care Med 1995; 151: 1472-80.

19.- WYSER C, STULZ P, SOLÈR M, TAMM M, MÜLLER-BRAND J, HABICHT J, et al. Prospective evaluation of an algorithm for the functional assessment of lung resection candidates. Am J Respir
Crit Care Med 1999; 159: 1450-6.

20.- WEBER K, en: Wasserman K. Principles of Exercise Testing and Interpretation. 3rd ed. Lippincott Williams \& Wilkins, Philadelphia, PA, USA, 1999.

21.- TJAHJA I E, REDDY H K, JANICKI J S, WEBER K T. Evolving role of cardiopulmonary exercise testing in cardiovascular disease. Clin Chest Med 1994; 15: 271-85.

22.- MYERS J, GULLESTAD L, VAGELOS R, DO D, BELLIN D, ROSS H, et al. Clinical, hemodynamic, and cardiopulmonary exercise test determinants of survival in patients referred for evaluation of heart failure. Ann Intern Med 1998; 129: 286-93.

23.- MANCINI D, EISEN H, KUSSMAUL W, MULL R, EDMUNDS L, WILSON J. Value of peak exercise oxygen consumption for optimal timing of cardiac transplantation in ambulatory patients with heart failure. Circulation 1991: 83; 778-86.

24.- STEVENSON L W, STEIMLE A E, FONAROW G, KERMANI M, KERMANI D, HAMILTON M A, et al. Improvement in exercise capacity of candidates awaiting heart transplantation. J Am Coll Cardiol 1995; 25: 163-70.

25.- O'NEILL J O, YOUNG J B, POTHIER C E, LAUER M S. Peak Oxygen Consumption as a Predictor of Death in Patients With Heart Failure Receiving b blockers. Circulation 2005; 111: 2313-8.

26.- FLEG J L, PIÑA I L, BALADY G J, CHAITMAN B R, FLETCHER B, LAVIE C, et al. Assessment of functional capacity in clinical and research applications. An advisory from the committee on exercise, rehabilitation, and prevention, council on clinical cardiology, American Heart Association. Circulation 2000; 102: 1591-97.

27.- ITOH H, TANIGUCHI K, KOIKE A, DOI M. Evaluation of severity of heart failure using ventilatory gas analysis. Circulation. 1990; 81(1 Suppl): II31-7.

28.- ARENA R, MYERS J, ASLAM S S, VARUGHESE E B, PEBERDY M A. Peak VO2 and VE/VCO2 slope in patients with heart failure: A prognostic comparison. American Heart Journal 2004; 147: 354-60.

29.- CHUA T P, PONIKOWSKI P, HARRINGTON D, ANKER S D, WEBB-PEPLOE K, CLARK A L, et al. Clinical correlates and prognostic significance of the ventilatory response to exercise in chronic heart failure. J Am Coll Cardiol 1997; 29: 1585-90.

30.- GITT A K, WASSERMAN K, KILKOWSKI C, KLEEMANN T, KILKOWSKI A, BANGERT M, et al. Exercise anaerobic threshold and ventilatory efficiency identify heart failure patients for high risk of early death. Circulation 2002; 106: 3079-84.

31.- ATS/ACCP Statement for Cardiopulmonary Exercise Testing. Am J Respir Crit Care Med 2003; 167: 21177.

Correspondencia a:

Dr. Rodrigo Soto Figueroa

Clínica Alemana de Santiago

Av. Manquehue $1410,8^{\circ}$ piso, Vitacura.

E-mail: rsoto955@hotmail.com 